\title{
BATATA CV PIRASSU COMO MATÉRIA-PRIMA INDUSTRIAL ${ }^{1}$
}

\author{
Emerson Loli Garcia ${ }^{2}$, Magali Leonel ${ }^{3}$, Ezequiel Lopes do Carmo ${ }^{4}$ \& Joaquim Gonçalves \\ de Pádua ${ }^{5}$
}

RESUMO: O mercado de amido vem crescendo e se aperfeiçoando nos últimos anos, levando a busca de produtos com características específicas que atendam as exigências industriais. Neste trabalho objetivou-se avaliar a composição centesimal dos tubérculos da batata cv Pirassu e suas potencialidades industriais. A batata cv Pirassu foi cultivada no município de Pouso Alegre, região serrana ao sul de Minas Gerais, com plantio em agosto e colheita em novembro de 2011. Os tubérculos foram analisados quanto à cor da casca, polpa e composição centesimal. O amido extraído foi analisado quanto à composição centesimal, teor de fósforo, amilose aparente, propriedades de pasta e propriedades térmicas. Os resultados obtidos mostraram que os teores de matéria seca, açúcar solúvel total e amido dos tubérculos são compatíveis para o processamento na forma de fritas tipo chips. O amido apresentou características importantes de viscosidade, tais como: resistência ao calor e atrito, boa viscosidade final e fraca organização granular, características importantes para a indústria alimentícia.

PALAVRAS-CHAVE: Solanum tuberosum L., propriedades reológicas, amido.

\section{POTATO TUBERS CV PIRASSU AS INDUSTRIAL RAW MATERIALS}

ABSTRACT: The starch market has been growing and improving in recent years, leading to a search for products with specific characteristics that meet the industrial requirements. This work aimed to evaluate the centesimal composition of potato tubers cv Pirassu and its industrial potential. The potato cv Pirassu was cultivated in Pouso Alegre, mountainous region south of Minas Gerais state, with planting season in August and harvest season in November, 2011. The tubers were analyzed for color, pulp and centesimal composition. The starch extract was analyzed for centesimal composition, phosphorus content, apparent amylose, pasting properties and thermal properties. The results showed that the content of dry matter, total soluble sugar and starch of tubers, are compatible for processing for potato chips. The starch of this cultivate showed important viscosity characteristics for food industry such as: heat resistance and friction, good final viscosity and low granular organization.

KEYWORDS: Solanum tuberosum L., rheological properties, starch.

\footnotetext{
${ }^{1}$ Parte da dissertação do primeiro autor intitulada: Composição dos tubérculos, extração e caracterização de amidos de diferentes cultivares de batata.

${ }_{2}^{2}$ Aluno do Programa de Pós-graduação em Agronomia: Energia na Agricultura - Centro de Raízes e Ámidos Tropicais (CERAT) Universidade Estadual Paulista, Botucatu, SP, Brasil. E-mail: emerson.cerat@gmail.com

${ }^{3}$ Orientadora e Diretora do Centro de Raízes e Amidos Tropicais (CERAT) - Universidade Estadual Paulista, Botucatu, SP, Brasil. Email:mleonel@cerat.unesp.br
}

\footnotetext{
${ }^{4}$ Prof. Dr. Instituto Federal do Mato Grosso (IFMT), campus Campo Novo do Parecis, Mato Grosso, MT, Brasil.

${ }^{5}$ Pesquisador da Embrapa de Pesquisa Agropecuária de Minas Gerais (EPAMIG) - Fazenda Experimental de Pouso Alegre, Pouso Alegre, MG, Brasil. CERAT - FCA/UNESP: Botucatu/SP - Brasil. Rua José Barbosa de Barros, 1780. CEP: 18610-307.
} 


\section{INTRODUÇÃO}

A batata (Solanum tuberosum L.) é a hortaliça de maior importância econômica no Brasil, sendo comercializada principalmente na forma in natura (ZORZELLA et al., 2003). Em nível mundial, a batata é plantada em mais de 125 países e consumida por mais de um bilhão de pessoas em todo o mundo (PASTORINI et al., 2003).

O Brasil ocupa o $19^{\circ}$ lugar no ranking da produção mundial de batatas, com uma área plantada em 2011 de 149,2 mil hectares e produção de 3,9 milhões de toneladas, sendo auto-suficiente na produção para atender o mercado da batata in natura. Por outro lado, o Brasil ainda depende da importação para atender o mercado de batata processada, principalmente da préfrita congelada.

A produção brasileira de batata assume um importante papel seja no aspecto econômico ou social, estando entre os dez principais produtos agrícolas do país, sendo cultivada principalmente nas regiões Sudeste e Sul, com Minas Gerais, Paraná e São Paulo, responsáveis por mais de $70 \%$ da produção brasileira (IBGE, 2013).

Contudo, no Brasil, a batata ainda é pouco explorada quanto sua forma de processamento, dentre elas pode-se citar a produção de amido, flocos, farinhas e a grande quantidade de produtos extrusados como: amidos prégelatinizados, farinhas instantâneas, snacks, sopas, macarrões, biscoitos (LEONEL, 2005).

Os tubérculos de batata são compostos por aproximadamente $76 \%$ de água, $17 \%$ de carboidratos, $2,0 \%$ de proteínas, $0,3 \%$ de açúcares redutores, $1,1 \%$ de cinzas, $25 \mathrm{mg} / 100 \mathrm{~g}$ de vitamina $\mathrm{C}$ e quantidades irrisórias de lipídios (SABLANI; MUJUMDAR, 2006).

Para Tester et al. (2004), os grânulos de amido são constituídos por dois polissacarídeos: amilopectina e amilose, sendo o componente principal de formação a glicose.

Segundo Leonel (2005), as propriedades funcionais dos amidos estão diretamente ligadas à distribuição e organização estrutural desses polissacarídeos dentro do grânulo. As indústrias alimentícias são as maiores consumidoras de amido, proporcionando novas oportunidades de mercado. O amido também pode ser utilizado em diversos outros segmentos (químicos, têxteis, petroquímicos), necessitando de cultivares com características específicas para cada propósito (SALAZAR; BUSCH, 2001).

Um fator importante no processamento de batata é o desenvolvimento de cultivares que atendam as exigências de qualidade de cada tipo de processamento, sendo os principais parâmetros divididos em: características agronômicas (produtividade, resistência ás pragas e doenças, adaptação climática, etc.) e composição química (teor de matéria seca, teor e açúcares e características da fração amilácea) (LEONEL et al., 2011).
Nesta linha, objetivou-se caracterizar tubérculos de batata cv Pirassu quanto à composição centesimal e cor, assim como, extrair e caracterizar o amido quanto às propriedades químicas, conteúdo de fósforo e propriedades reológicas, visando o potencial de aplicabilidade industrial da cultivar.

\section{MATERIAL E MÉTODOS}

\subsection{Localização do experimento}

A batata cv Pirassu foi cultivada no município de Pouso Alegre, região serrana ao sul de Minas Gerais. A região apresenta um clima tropical de altitude, as chuvas de verão são mais abundantes, e no inverno as frentes frias, vindas do Pólo Sul, podem provocar o fenômeno da geada, apresentando temperatura média anual de $18,2^{\circ} \mathrm{C}$. A precipitação pluvial média anual no município está em torno de $1642,2 \mathrm{~mm}$. O solo é do tipo Latossolo Vermelho-Amarelo, textura argilosa com boa profundidade. O plantio ocorreu em 08 de agosto e a colheita em 28 de novembro de 2011.

\subsection{Análise dos tubérculos}

Os tubérculos colhidos e lavados foram caracterizados quanto ao teor de umidade, lipídios, proteínas, cinzas, fibra bruta, açúcares totais, amido, acidez titulável e pH (AOAC, 2007). Todas as análises foram realizadas em triplicatas.

A cor da casca e da polpa foi avaliada em colorímetro Minolta CR-400. Os resultados foram expressos em valores $\mathrm{L}^{*}, \mathrm{a}^{*} \mathrm{e} \mathrm{b}^{*}$, onde os valores de $\mathrm{L}^{*}$ (luminosidade ou brilho) variam do preto (0) ao branco (100), os valores do croma $a^{*}$ variam do verde $(-60)$ ao vermelho $(+60)$ e os valores do croma $b^{*}$ variam do azul ao amarelo, ou seja, de -60 a +60 , respectivamente.

Para extração do amido, os tubérculos foram lavados e desintegrados na presença de água gelada em liquidificador industrial. A suspensão obtida foi passada por peneiras de abertura de $0,250 \mathrm{~mm}$ e $0,088 \mathrm{~mm}$, respectivamente. $\mathrm{O}$ bagaço retido nas peneiras voltou novamente ao liquidificador para retirada do amido residual. Após a decantação do amido, houve a lavagem para retirada de impurezas, sendo, seco em estufa de circulação de ar forçado à temperatura de $40^{\circ} \mathrm{C}$.

\subsection{Caracterização do amido}

$\mathrm{O}$ amido foi caracterizado quanto aos teores de umidade, proteína, amido, lipídios, açúcares totais, fibra, $\mathrm{pH}$ e acidez titulável (AOAC, 2007). O teor de amilose aparente foi determinado segundo metodologia descrita por ISO-6647 (INTERNATIONAL ORGANIZATION FOR STANDARDIZATION, 1987) e o teor de fósforo determinado conforme metodologia descrita por Malavolta et al. (1974).

As propriedades de pasta foram determinadas utilizando o Analisador de Viscosidade Rápida (RVA). A amostra foi pesada conforme umidade e adicionado aproximadamente $25 \mathrm{~mL}$ de água. As características avaliadas foram temperatura de pasta, viscosidade 
máxima (pico), queda de viscosidade, viscosidade final e tendência a retrogradação. Os parâmetros foram avaliados pelo software Thermocline for Windows, versão 2.2 , utilizando o perfil de temperatura e tempo $2 \mathrm{Std} 2$.

As propriedades térmicas foram analisadas utilizando um Calorímetro Diferencial de Varredura, equipado com um intracooler e um software de análise térmica (Pyris 1Perkin Elmer, EUA) para determinação das temperaturas de gelatinização e retrogradação. Aproximadamente 2 mg (b.s.) de amido foram pesados em cápsulas de alumínio e $6 \mu \mathrm{L}$ de água deionizada foram adicionados, em seguida seladas. As suspensões de amido foram deixadas em equilíbrio por $1 \mathrm{~h}$ à temperatura ambiente. As amostras foram aquecidas de 25 a $100^{\circ} \mathrm{C}$ a uma taxa de $10^{\circ} \mathrm{C}$ min-1. Uma cápsula vazia foi utilizada como referência. As análises de retrogradação foram realizadas após armazenamento dos recipientes padrões contendo as amostras gelatinizadas a $4^{\circ} \mathrm{C}$ por 14 dias e novamente analisadas utilizando as mesmas condições anteriores. Os parâmetros avaliados foram: Temperatura inicial (T0), pico (Tp), final (Tf), variação de temperatura $(\Delta \mathrm{T})$ e variação de entalpia $(\Delta \mathrm{H})$ e tendência a retrogradação (\%R).

\section{RESULTADOS E DISCUSSÃO}

\subsection{Análise da cor: casca e polpa}

A cv Pirassu (Tabela 1) apresentou luminosidade para o tubérculo inferior à relatada por Caetano (2006), os valores encontrados pelo autor para diferentes cultivares de batata variaram entre 61,71 a 84,67. Para os parâmetros $a^{*}$ e $b^{*}$, a cultivar apresentou, intensa presença dos componentes de cor vermelha e amarela na casca. Segundo Fernandes et al. (2010), no momento da compra, o consumidor, escolhe prioritariamente a qualidade da batata por características visuais, tais como: cor e brilho da casca. De acordo com Silva et al. (2006), a película amarela dos tubérculos, é uma importante característica, uma vez que os consumidores preferem batatas de tonalidade clara em relação as mais escuras, pois atribuem a tonalidade clara à batata recém-colhida.

Para a polpa, a cultivar apresentou polpa clara com presença dos componentes de cor verde e amarela. De acordo com Pereira et al. (2005), cultivares que possuem polpa amarela são melhores para serem processadas através do cozimento.

\section{Tabela 1 - Média \pm desvio padrão da caracterização da cor da casca e da polpa da cultivar Pirassu.}

\begin{tabular}{ccc}
\hline Parâmetro & Casca & Polpa \\
\hline $\mathrm{L}^{*}$ & $53,50( \pm 1,23)$ & $76,61( \pm 0,21)$ \\
$\mathrm{a}^{*}$ & $12,88( \pm 0,63)$ & $-1,28( \pm 0,01)$ \\
$\mathrm{b}^{*}$ & $22,48( \pm 0,48)$ & $28,34( \pm 1,27)$ \\
\hline
\end{tabular}

L* - luminosidade; $a^{*}$ - variação da cor vermelha $\left(+a^{*}\right)$ ao verde $\left(-a^{*}\right)$; b* - variação da cor amarela $\left(+b^{*}\right)$ ao azul $\left(-b^{*}\right)$.

\subsection{COMPOSIÇÃO CENTESIMAL}

Os resultados para a composição centesimal dos tubérculos e do amido são demonstrados na Tabela 2. A cv Pirassu apresentou 19,6 \% de matéria seca, que segundo a classificação de Cacace et al. (1994), é um bom teor de matéria seca para batatas destinadas ao processamento na forma de palito pré-frito.

Segundo Zorzella et al. (2003), tubérculos com elevado teor de matéria seca podem produzir fritas com excelente qualidade, por minimizar perdas de água, consequentemente, reduzindo a absorção de gordura no momento da fritura, favorecendo o rendimento e o sabor característico da batata frita.

O teor de amido observado foi de 16,68\%, dentro da média de $16 \%$ do peso in natura citado por Rodrigues Robles (2003) para batatas. Além disso, Kita (2002) estudando a relação entre a textura e o conteúdo de amido das batatas voltadas para a indústria de fritas, concluiu que para garantir maior crocância, o teor de amido deve ser superior a $15 \%$.

De acordo com Borruey et al. (2000), para se obter fritas de excelente qualidade o conteúdo de açúcares nos tubérculos deve ser inferior a $0,15 \%$ para fritas tipo Chips e $0,25 \%$ para as processadas na forma de palito, sendo a cultivar Pirassu indicada para o processamento na forma de frito tipo Chips, apresentando um teor de $0,12 \%$ de açúcares em base úmida.

Tabela 2 - Média \pm desvio padrão da composição centesimal do tubérculo e do amido da cultivar Pirassu.

\begin{tabular}{lcc}
\hline \multicolumn{1}{c}{$\begin{array}{c}\text { Parâmetro } \\
\text { g. } 100 \mathrm{~g}^{-1}\end{array}$} & $\begin{array}{c}\text { Tubérculo } \\
\text { Base úmida }\end{array}$ & Amido \\
\hline Umidade & $80,4( \pm 0,69)$ & $10,93( \pm 0,15)$ \\
Amido & $16,68( \pm 0,28)$ & $92,98( \pm 0,05)$ \\
Cinzas & $0,40( \pm 0,05)$ & $0,27( \pm 0,02)$ \\
Proteína & $1,95( \pm 0,27)$ & $0,25( \pm 0,02)$ \\
Lipídios & $0,06( \pm 0,08)$ & $0,25( \pm 0,03)$ \\
Fibra Alimentar & $0,61( \pm 0,15)$ & $0,30( \pm 0,03)$ \\
Açúcares Totais & $0,12( \pm 0,10)$ & $0,05( \pm 0,01)$ \\
\hline pH & $6,36( \pm 0,08)$ & $7,44( \pm 0,02)$ \\
Ac. Titulável & $10,4( \pm 0,10)$ & $0,26( \pm 0,05)$ \\
\hline
\end{tabular}

Segundo Vendrusculo (1998), o limite estabelecido na literatura quanto ao teor de açúcares é quase consensual, sendo que para tubérculos destinados à fritura ficam situados entre 0,2 a $0,3 \%$ em base úmida, sendo os açúcares fator limitante na depreciação da cor (MÁRQUEZ; AÑON, 1986), baixos teores de açúcares proporcionam um produto final muito claro e elevados teores provocam a reação de Maillard, levando ao escurecimento do produto durante a fritura e também a não aceitação pelo consumidor (ZORZELLA et al., 2003).

O valor médio de cinzas encontrado para cv. Pirassu foi de $0,4 \%$, estando próximo à faixa de 0,44 a $1,90 \%$ relatado por Trindade (1994). O conteúdo de proteína 
apresentado para a cv Pirassu foi de 1,95\% estando de acordo com os resultados encontrados por Evangelista et al. (2011), os autores, encontraram teores de proteína variando entre 1,42 a $2,06 \%$ para diversas cultivares de batata.

O teor médio de lipídios encontrado foi de $0,06 \%$, confirmando traços deste componente, estando de acordo com a Tabela Brasileira de Composição de Alimentos (TACO-UNICAMP, 2006). Teores semelhantes foram obtidos por Bregagnoli (2006), o autor encontrou conteúdo de lipídios para as cultivares Atlantic e Asterix variando entre 0,07 a $0,08 \%$ e 0,06 a $0,11 \%$, respectivamente, corroborando com os dados relatados neste trabalho.

O amido é constituído praticamente de carboidratos, no entanto, substâncias como lipídios, proteínas e cinzas estão presentes em sua composição. A quantidade desses componentes depende da composição da planta e dos métodos de extração e purificação.

Os resultados obtidos para o amido da cv Pirassu mostraram baixo teor de componentes não amido, sendo, a pureza do amido relacionada com baixos teores destes constituintes (Tabela 2). Segundo Mestres (1996), teores de proteína superior a $0,5 \%$ podem interferir nas propriedades funcionais do amido. De acordo com Peroni (2003), a pureza do amido é um importante parâmetro de qualidade, facilitando as aplicações industriais. Os valores encontrados para $\mathrm{pH}$ e acidez titulável estão de acordo com a legislação brasileira (BRASIL, 2005).

\subsection{Teor de amilose aparente}

O teor de amilose está diretamente relacionado com a constituição estrutural das regiões cristalinas e amorfas do grânulo (GALLANT et al., 1982), afetando as propriedades funcionais de gelatinização, retrogradação e a susceptibilidade à hidrólise enzimática do amido (GÉRARD et al., 2001). O teor encontrado para cv Pirassu foi de 35,44\%. Segundo Srichuwong et al. (2005) a amilose representa em média 15 a $30 \%$ do amido e esse conteúdo pode variar de acordo com a fonte botânica, maturidade fisiológica e condições sazonais do cultivo (CEREDA et al., 2002).

Segundo Guerreiro (2002) quando se determina o teor de amilose aparente em amidos pode ocorrer à quantificação de componentes intermediários do grânulo e/ou cadeias laterais de amilopectina como sendo amilose, segundo Jane et al. (1999) tais compostos podem interagir com o iodo superestimando o teor de amilose presente. Os teores de amilose podem sofrer grandes variações devido a diferentes metodologias de determinação, variedade ou idade da planta (RECHSTEINER, 2009).

\subsection{Teor de minerais}

Dentre os minerais encontrados na batata, o de maior contribuição para aplicação industrial é sem dúvida o fósforo. O fósforo é encontrado em raízes e tubérculos ligado covalentemente ao amido na forma de monoéster fosfato, contribuindo para aumentar a claridade de pasta, viscosidade, aumento da capacidade de ligação com a água e poder de inchamento além de contribuir para diminuição da temperatura de gelatinização e a taxa de retrogradação (SINGH et al., 2003; HOOVER, 2001).

O teor de fósforo observado no amido da cv Pirassu foi de $0,030 \%$, teor este, inferior ao relatado por Franco et al. (2002) para amido de batata $(0,09 \%)$. Contudo, teores próximos foram relatados por Noda et al. (2007), os quais, analisando diferentes cultivares de batata e os efeitos sobre as propriedades do amido, observaram teores de fósforo variando entre 0,031 a 0,124\%. Apesar do baixo teor de fósforo presente no amido da cv Pirassu, este, pode interferir positivamente nas propriedades funcionais, portanto, uma importante vantagem para muitas aplicações industriais.

\subsection{Propriedades de pasta}

As mudanças que ocorrem nos grânulos durante a gelatinização e a retrogradação são os principais determinantes do comportamento de pasta dos amidos, as quais são medidas principalmente pelas mudanças de viscosidade durante e após o aquecimento das suspensões de amido (LUSTOSA et al., 2009), as mudanças, são afetadas principalmente pelos teores de amilose, lipídios, fósforo e pela distribuição dos comprimentos de cadeias ramificadas da amilopectina (TESTER; MORRISON, 1990). As propriedades de pasta do amido da cv Pirassu são demonstradas na Tabela 3 .

Os resultados obtidos no presente trabalho para as propriedades de pasta foram superiores aos observados por Kaur et al. (2007), estudando diferentes amidos de batata, observaram viscosidade inicial variando de 2351 a $3606 \mathrm{cP}$; pico de viscosidade de 4145 a $7229 \mathrm{cP}$; quebra de viscosidade de 1505 a $4490 \mathrm{cP}$; viscosidade final de 1950 a $3204 \mathrm{cP}$ e temperatura de empastamento de 64,5 a $69,4^{\circ} \mathrm{C}$.

\begin{tabular}{lc} 
Tabela 3 - $\begin{array}{c}\text { Média } \pm \text { desvio padrão das } \\
\text { propriedades de pasta do amido } \\
\text { da cultivar Pirassu. }\end{array}$ \\
\hline \multicolumn{1}{c}{ Parâmetros } & RVU \\
\hline Viscosidade Máxima & $778,38( \pm 1,12)$ \\
Quebra de Viscosidade & $509,71( \pm 1,05)$ \\
Viscosidade Final & $377,30( \pm 4,79)$ \\
Tend. Retrogradação & $108,63( \pm 6,57)$ \\
\hline Tempo Pico $(\min )$ & $5,57( \pm 0,03)$ \\
Temp. de Pasta $\left({ }^{\circ} \mathrm{C}\right)$ & $66,75( \pm 0,04)$ \\
\hline
\end{tabular}

$1 \mathrm{RVU}=12 \mathrm{cP}$.

Contudo, resultados semelhantes foram observados por Leonel e Sarmento (2002) para amidos de batata. Importantes características foram observadas para este amido, tais como: baixa temperatura de empastamento, resistência ao calor e ao atrito, bom nível de viscosidade final, fraca organização granular, indicando fácil cozimento.

Não se pode descartar um amido por sua viscosidade característica de pasta, pois de acordo com o tipo de 
alimento uma viscosidade maior ou menor será desejável. Uma sopa pronta, por exemplo, não deve apresentar viscosidade final muito elevada, o que causaria uma sensação desagradável. Mas, para o recheio de tortas, uma maior viscosidade é desejável, entretanto, deve se considerar que o processamento industrial reduz a viscosidade, e essa queda deve ser considerada no cálculo de viscosidade desejada ao produto final (DAIUTO, 2005).

\subsection{Propriedades térmicas}

Através do conhecimento das propriedades térmicas, pode-se determinar a funcionalidade específica de cada amido, portanto, sua utilização na indústria de alimentos. Os resultados obtidos para as propriedades térmicas da cv Pirassu (Tabela 4) corroboram com os dados observados por Kaur et al. (2007) que, analisando as propriedades de gelatinização de amidos de diferentes cultivares de batata observaram $\mathrm{T}_{0}$ variando de 58,3 a $62,21^{\circ} \mathrm{C} ; \mathrm{T}_{\mathrm{p}}$ de 61,6 a $66,75^{\circ} \mathrm{C}, \mathrm{T}_{\mathrm{f}}$ de 66,63 a $76,98^{\circ} \mathrm{C}$ e $\Delta \mathrm{H}$ de 10,6 a 16,9J.g ${ }^{-1}$. Para Thomas e Atwell (1999), as temperaturas de gelatinização para tubérculos de batata ocorrem entre $58-65^{\circ} \mathrm{C}$, sendo que os resultados encontrados para cv Pirassu, corroboram para tal afirmação.

Comparando-se as temperaturas de gelatinização com as temperaturas de pasta observa-se que as temperaturas iniciais de gelatinização foram menores que as encontradas para as temperaturas de pasta, o mesmo efeito foi observado por Pérez et al. (1998) e Jane et al. (1999), sendo essa diferença característica do tipo de equipamento utilizado. Enquanto o DSC detecta a hora que os primeiros grânulos começam a se desorganizar o RVA detecta os primeiros acréscimos de viscosidade.

Tabela 4 - Média \pm desvio padrão das propriedades térmicas de gelatinização e retrogradação do amido da cultivar Pirassu.

Gelatinização

\begin{tabular}{ccccc}
\hline $\mathrm{T}_{0}$ & $\mathrm{~T}_{\mathrm{p}}$ & $\mathrm{T}_{\mathrm{f}}$ & $\Delta \mathrm{T}$ & $\begin{array}{c}\Delta \mathrm{H} \\
\left(\mathrm{J} . \mathrm{g}^{-1}\right)\end{array}$ \\
\hline 63,345 & 66,505 & 70,760 & 7,415 & 14,962 \\
$( \pm 0,05)$ & $( \pm 0,10)$ & $( \pm 0,17)$ & $( \pm 0,13)$ & $( \pm 0,50)$ \\
\hline \multicolumn{5}{c}{ Retrogradação } \\
\hline $\mathrm{T}_{0}$ & $\mathrm{~T}_{\mathrm{p}}$ & $\mathrm{T}_{\mathrm{f}}$ & $\Delta \mathrm{H}$ & $\% \mathrm{R}$ \\
\hline 47,93 & 58,50 & 68,32 & 7,01 & 46,87 \\
$( \pm 0,05)$ & $( \pm 0,09)$ & $( \pm 0,29)$ & $( \pm 0,14)$ & $( \pm 0,66)$ \\
\hline
\end{tabular}

$\mathrm{T}_{0}, \mathrm{~T}_{\mathrm{p}}, \mathrm{T}_{\mathrm{f}}, \Delta \mathrm{H}$ e $\% \mathrm{R}=$ respectivamente, temperatura inicial, pico, final, variação de temperatura, variação de entalpia e tendência a retrogradação.

Avaliando as propriedades térmicas de retrogradação observa-se que os valores dos parâmetros $\mathrm{T}_{0}, \mathrm{~T}_{\mathrm{p}}, \mathrm{T}_{\mathrm{f}}, \Delta \mathrm{H}$ foram inferiores aos apresentados nas propriedades térmicas de gelatinização, sendo o mesmo comportamento observado por Karim et al. (2000).

Para os autores Karim et al. (2000) os amidos retrogradados podem apresentar valores de entalpia de até $60-80 \%$ menores que os obtidos para os amidos nativos, os autores ainda, relatam que na retrogradação, o gel sofre transformações que resultam em uma estrutura parcialmente cristalina, diferente da estrutura presente no grânulo de amido nativo.

Segundo Franco et al. (2002) a batata apresenta baixa tendência em retrogradar, atribuindo esta ocorrência a alta massa molecular da fração amilose e a presença dos grupos fosfatos ligados ao amido, sendo o mesmo comportamento observado por Acquarone e Rao (2003), as ligações inter/intra molecular em posições aleatórias entre as cadeias de amilopectina com o fósforo provocam maior estabilidade ao grânulo.

Diante do exposto, é possível verificar que a cultivar Pirassu apresentou elevada tendência em retrogradar $(46,87 \%)$, essa ocorrência pode ser devida ao baixo conteúdo de fósforo ligado covalentemente ao grânulo de amido, acarretando assim, em um elevado potencial em retrogradar e elevada energia necessária para romper as ligações das duplas hélices.

\section{CONCLUSÃO}

A cv Pirassu apresenta características compatíveis para o processamento na forma de chips. $\mathrm{O}$ amido apresentou elevado teor de amilose, baixo teor de fósforo, baixa temperatura de empastamento, resistência ao calor e ao atrito e elevado potencial de retrogradação.

\section{REFERÊNCIAS}

ACQUARONE, V. M.; RAO, M. A. Influence of sucrose on the rheology and granule size of cross-linked waxy maize starch dispersions heated at two temperatures. Carbohydrates Polymers, Oxford, v. 51, n. 4 p. 451-458, Mar. 2003. Disponível em: $<$ http://www.sciencedirect.com/science/article/pii/S0144 861702002175>. Acesso em: 05 jul. 2013.

\section{ASSOCIATION OF OFFICIAL ANALYTICAL CHEMISTS INTERNATIONAL - AOAC -} International: Official methods of analysis of AOAC International. $18^{\text {th }}$ edition - Gaithersburg. Current Through Revision 2, 2007.

BORRUEY, A. et al. Calidade industrial y culinária de las variedades de patata. In: PASCUALENA J.; RITTER, E. Actas del congreso iberoamericano de investigación y desarrollo en patata, Editorial VitoriaGastéis, España, p.1-15, 2000.

BRASIL. Agência Nacional de Vigilância Sanitária. Resolução no 263. Aprova o "Regulamento técnico para produtos de cereais, amidos, farinhas e farelos". Diário Oficial da União, Poder Executivo, Brasília, DF, 23 set. 2005.

BREGAGNOLI, M. Qualidade e produtividade de cultivares de batata para indústria sob diferentes adubações. 2006. 141 f. Tese (Doutorado em Agronomia/Fitotecnia) -Escola Superior de Agricultura Luiz de Queiroz, Universidade de São Paulo, Piracicaba, 2006. 
CACACE, J. E. et al. Evaluation of potato cooking quality in Argentina. American Potato Journal, Orono, v. $71, \quad$ n. 3, p. 145-153, 1994. DOI: 10.1007/BF02849049. Disponível em: $<$ http://link.springer.com/article/10.1007/BF02849049 . Acesso em: 10 set. 2013.

CAETANO, D. Inibição do escurecimento na produção de farinha de batata (Solanum tuberosum L.) utilizando secador solar tipo túnel. 2006. $96 \mathrm{f}$. Dissertação (Mestrado em Ciências dos Alimentos) Universidade Federal de Lavras, Lavras, 2006.

CEREDA, M. P. et al. Propriedades gerais do amido. In: CEREDA, M. P. et al. Culturas de tuberosas amiláceas latino americanas. São Paulo: Fundação Cargill, p. 204, v.1, 2002.

DAIUTO, E. R. Características de féculas de tuberosas e suas relações com resistência dos géis sob condições de estresse aplicada na industrialização de alimentos. 2005. 146 f. Tese (Doutorado em Agronomia/Energia na Agricultura) -Faculdade de Ciências Agronômicas, Universidade Estadual Paulista, Botucatu, 2005.

EVANGELISTA, R. M. et al. Qualidade nutricional e esverdeamento pós-colheita de tubérculos de cultivares de batata. Pesquisa Agropecuária Brasileira, Brasília, DF, v. 46, n. 8, p. 953-960, ago. 2011. Disponível em: $<$ http://seer.sct.embrapa.br/index.php/pab/article/view/10 181>. Acesso em: 10 jul. 2013.

FERNANDES, A. M. et al. Qualidade físico-química e de fritura de tubérculos de cultivares de batata na safra de inverno. Horticultura Brasileira, Brasília, DF, v. 28, n. 3, p. 299-304, July/Sept. 2010. Disponível em: $<$ http://dx.doi.org/10.1590/S0102-

$05362010000300010>$. Acesso em: 08 jun. 2013.

FRANCO, C. M. L. et al. Structural and functional characteristics of selected soft wheat starches. Cereal Chemistry, Saint Paul, v. 79, n. 2, p. 243-248, Mar./Apr. 2002. Disponível em: <http://dx.doi.org/10.1094/CCHEM.2002.79.2.243>. Acesso em: 22 ago. 2013.

GALLANT, D. J. et al. On ultrastructural and nutritional aspects of some tropical tuber starches. Starch/Stärke, Weinheim, v. 34, n. 8, p. 255-262, 1982. Disponível em: <http://dx.doi.org/10.1002/star.19820340803>. Acesso em: 05 abr. 2013.

GÉRARD, C.; BARRON, C. P.; PLANCHOT, V. Amylose determinations in genetically modified starches. Carbohydrate Polymers, Oxford, v. 44, n. 1, p. 19-27, Jan. 2001. Disponível em: <http://dx.doi.org/10.1016/S0144-8617(00)00194-6>. Acesso em: 31 maio 2013.
GUERREIRO, L. M. R. Avaliação de amidos nativos em condições de estresse adaptados ao processamento de alimentos. 2002. 180 p. Dissertação (Mestrado em Agronomia/Energia na Agricultura)- Faculdade de Ciências Agronômicas, Universidade Estadual Paulista, Botucatu, 2002.

HOOVER, R. Composition, molecular structure, and physicochemical properties of tuber and root starches: a review. Carbohydrate Polymers, Oxford, v. 45, n. 3, p. 253-267, July 2001. Disponível em: <http://dx.doi.org/10.1016/S0144-8617(00)00260-5>. Acesso em: 15 abr. 2013.

IBGE. Levantamento Sistemático da Produção Agrícola. Rio de Janeiro, v. 26, n. 10, nov. 2013. Disponível em: $<$ http://www.ibge.gov.br/home/estatistica/indicadores/ag ropecuaria/lspa/lspa_201310.pdf $\geq$. Acesso em: $30 \mathrm{de}$ nov. 2013.

INTERNATIONAL ORGANIZATION FOR STANDARDIZATION. ISO 6647: norme internacionale: riz determination de la teneur en amylose. Geneva, 4p, 1987.

JANE, J. et al. Effects of amylopectin branch length and amylose content on the gelatinization and pasting properties of starch. Cereal Chemistry, Saint Paul, v. 76, n. 5, p. 629-637, Sept./Oct. 1999. Disponível em: http://dx.doi.org/10.1094/CCHEM.1999.76.5.629.

Acesso em: 22 jan. 2013.

KARIM, A.; NORZIAH, M. H.; SEOW, C. C. Methods for the study of starch retrogradation. Food Chemistry, London, v. 71, n. 1, p. 9-36, Oct. 2000. Disponível em: <http://dx.doi.org/10.1016/S0308-8146(00)00130-8>. Acesso em: 20 jan. 2013.

KAUR, A. et al. Physicochemical, thermal and pasting properties of starches separated from different potato cultivars grown at different locations. Food Chemistry, London, v. 101, n. 2, p. 643-651, 2007. Disponível em: <http://dx.doi.org/10.1016/j.foodchem.2006.01.054>. Acesso em: 17 ago. 2013.

KITA, A. The influence of potato chemical composition on crisp texture. Food Chemistry, London, v. 76, n. 2, p. 173-179, 2002. Disponível em: <http://dx.doi.org/10.1016/S0308-8146(01)00260-6>. Acesso em: 15 set. 2013.

LEONEL, M.; PADUA, J. G.; DUARTE FILHO, J. Tecnologias de processamento de batata. In: ZAMBOLIM, L. (Org.). Produção integrada de batata. Visconde do Rio Branco: Suprema Gráfica e Editora, 2011. v. 2, p. 269-289.

LEONEL, M. Processamento de batata: fécula, flocos, produtos de extrusão. In: SEMINÁRIO MINEIRO SOBRE PROCESSAMENTO DE BATATAS, 2005, Pouso Alegre. Anais... Pouso Alegre: EPAMIG, 2005. 1 CD-ROM. 
LUSTOSA, B. H. B. et al. Produção de farinha instantânea de mandioca: efeito das condições de extrusão sobre as propriedades térmicas e de pasta. Acta Scientiarum Technology, Maringá, v. 31, n. 2,

p. 231-238, 2009. Disponível em: <http://periodicos.uem.br/ojs/index.php/ActaSciTechnol/ article/view/892/892>. Acesso em: 07 set. 2013.

MALAVOLTA, E. et al. Nutrição mineral e adubação de plantas cultivadas. São Paulo: Pioneira, p. 727, 1974.

MÁRQUEZ, G.; AÑON, M.C. Influence of reducing sugars and amino acids in the color of development of fried potatoes. Journal of Food Science, Chicago, v. 51, n. 1 , p. 157-160, 1986. Disponível em: $<$ http://dx.doi.org/10.1111/j.1365-

2621.1986.tb10859.x>. Acesso em: 27 ago. 2013.

MESTRES, C. Los estados fisicos del amidon. In: CONFERENCIA INTERNACIONAL DEL ALMIDON, 1996, Quito. Anais... Quito: Escuela Politécnica Nacional Instituto de Investigación Tecnológica, p.1-16, 1996.

NODA, T. et al. Starch phosphorus content in potato (Solanum tuberosum L.) cultivars and its effect on other starch properties. Carbohydrate Polymers, Oxford, v. 68, n. 4, p. 793-796, Apr. 2007. Disponível em: <http://dx.doi.org/10.1016/j.carbpol.2006.08.005>. Acesso em: 21 jan. 2013.

PASTORINI, L.H. et al. Produção e teor de carboidratos não estruturais em tubérculos de batata obtidos em duas épocas de plantio. Horticultura Brasileira, Brasília, DF, v. 21, n. 4, p. 660-665, out./dez. 2003. Disponível em:

$<$ http://www.horticulturabrasileira.com.br/index.php?opt ion=com_content $\&$ view $=$ article $\&$ id $=104 \&$ Itemid $=28$

\&artigo=http://www.horticulturabrasileira.com. br/images/stories/21_4/200321417.pdf>. Acesso em: 30 mar. 2013.

PEREIRA, E. M. S.; LUZ, J. M. Q.; MOURA, C. C. A batata e seus benefícios nutricionais. Uberlândia: EDUFU, p.60, 2005.

PÉREZ, E. E.; BREENE, W. M.; BAHNASSEY, Y. A. Variations in the gelatinization profiles of cassava, sagu and arrowroot native starches as measured with different thermal and mechanical methods. Starch/Stärke, Weinheim, v. 50, n. 2/3, p. 70-72, 1998. Disponível em: $<$ http://dx.doi.org/10.1002/(SICI)1521-

379X(199803)50:2/3<70:AID-STAR70>3.0.CO;2-U>. Acesso em: 16 jun. 2013.

PERONI, F. H. G. Características estruturais e físicoquímicas de amidos obtidos de obtidos de diferentes fontes botânicas. 2003. $118 \mathrm{f}$. Dissertação (Mestrado em Engenharia e Ciência de Alimentos) - Instituto de Biociências Letras e Ciências Exatas, Universidade Estadual Paulista, São José do Rio Preto, 2003.
RECHSTEINER, M. S. Desenvolvimento de Amidos Fosfatados de Batata-doce e Mandioca e Aplicação como Substitutos de Gordura em Sorvete. 2009. 167f. Tese (Doutorado em Agronomia - Energia na Agricultura) - Faculdade de Ciências Agronômicas da UNESP, Botucatu, 2009.

RODRIGUES ROBLES, W. G. Dióxido de carbono via fertirrigação em batateira (Solanum tuberosum L.) sob condições de campo. 2003. 160 f. Tese (Doutorado em Agronomia/Irrigação e Drenagem) -Escola Superior de Agricultura "Luiz de Queiroz", Universidade de São Paulo, Piracicaba, 2003.

SABLANI, S. S.; MUJUMDAR, A. S. Drying of potato, sweet potato, and other roots: handbook of industrial drying. $3^{\text {rd }}$ ed. London: Taylor \& Francis, p. 647-646, 2006.

SALAZAR, M.; BUSCH, L. Standards and strategies in the michigan potato industry. East Lansing: Michigan Agricultural Experiment Station, p.16, 2001. (Research Report, 576).

SILVA, G. O. et al. Características físico-químicas de amidos modificados de grau alimentício comercializados no Brasil. Ciência e Tecnologia de Alimentos, Campinas, v. 26, n. 1, p. 188-197, jan./mar. 2006. Disponível em: <http://dx.doi.org/10.1590/S0101$20612006000100030 \geq$. Acesso em: 01 ago. 2013.

SINGH, N. et al. Morphological, thermal and rheological properties of starches from different botanical sources. Food Chemistry, London, v. 81, n. 2, p. 219-231, May 2003. Disponível em: <http://dx.doi.org/10.1016/S03088146(02)00416-8>. Acesso em: 09 mar. 2013.

SRICHUWONG, S. et al. Starches from different botanical sources I: contribution of amylopectin fine structure to thermal properties and enzyme digestibility. Carbohydrate Polymers, Oxford, v. 60, n. 4, p. 529538, June 2005. Disponível em: <http://dx.doi.org/10.1016/j.carbpol.2005.03.004>. Acesso em: 11 jul. 2013.

TACO - Tabela Brasileira de Composição de Alimentos. 2ed. Campinas: NEPA-UNICAMP, 2006.

TESTER, R.; KARKALAS, J.; QI, X. Starch: composition, fine structure and architecture. Journal of Cereal Science, Glasgow, v. 39, n. 2, p. 151-165, Mar. $2004 . \quad$ Disponível em: $<$ http://dx.doi.org/10.1016/j.jcs.2003.12.001>. Acesso em: 13 jun. 2013.

TESTER, R. F.; MORRISON, W. R. Swelling and gelatinization of cereal starches. I. Effects of amylopectin, amylose and lipids. Cereal Chemistry, Saint Paul, v. 67, n. 6, p. 551-557, 1990. Disponível em: $<$ http://www.aaccnet.org/publications/cc/backissues/199 0/Documents/67_551.pdf>. Acesso em: 23 maio 2013. 
THOMAS, D. J.; ATWELL, W. A. Starches: practical guides for the food industry. Minnesota: Eagan Press, 1999.94 p.

TRINDADE, J. L. F. Caracterização de algumas variedades de batata do Município de Contenda-PR e indicações quanto ao uso doméstico e fins tecnológicos. 1994. 91 f. Dissertação (Mestrado em Tecnologia de Alimentos) - Universidade Federal do Paraná, Curitiba, 1994.

VENDRUSCOLO, J.L. Avaliação e melhoria das qualidades tecnológicas e sensoriais de genótipos de batata (Solanum tuberosum L.) para a industrialização e consumo de mesa. Pelotas, CPACT/EMBRAPA, 1998. p. 6 - Subprojeto de Pesquisa $\mathrm{n}^{\mathrm{o}} \quad 0.5 .0 .99 .080 .05$ Sistema Embrapa de Planejamento.

ZORZELLA, C. A. et al. Caracterização física, química e sensorial de genótipos de batata processados na forma chips. Brazilian Journal Food Technology, Campinas, v. 6, p. 15-24, 2003. Disponível em: <http://bjft.ital.sp.gov.br/artigos/html/busca/PDF/v6nu10 8a.pdf>. Acesso em: 27 ago. 2013. 